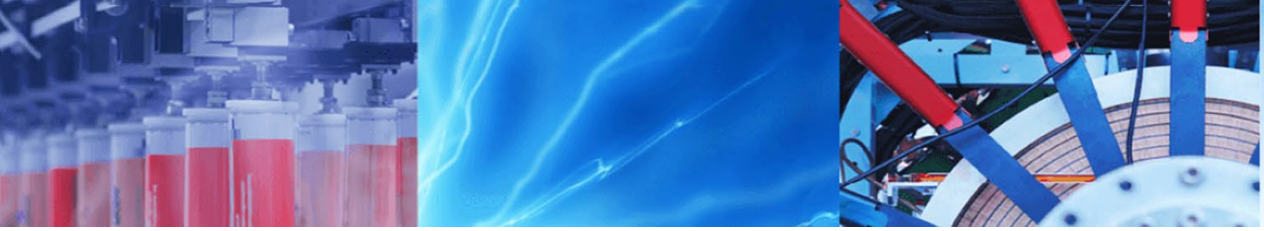

Research Article

\title{
Influence of chamber design on the gas sensing performance of graphene field-effect-transistor
}

\author{
Lorenzo Lopez Jr. ${ }^{1,2,3} \cdot$ Vernalyn Copa ${ }^{1,2,3} \cdot$ Takeshi Hayasaka $^{3} \cdot$ Maria Angela Faustino-Lopez ${ }^{1,2} \cdot$ Yichuan Wu $^{3}$. \\ Huiliang Liu ${ }^{3} \cdot$ Yumeng Liu ${ }^{3}$. Elmer Estacio ${ }^{1,2} \cdot$ Armando Somintac $^{1,2} \cdot$ Liwei Lin $^{3} \cdot$ Arnel Salvador $^{1,2}$
}

Received: 1 November 2019 / Accepted: 2 April 2020 / Published online: 8 June 2020

(c) Springer Nature Switzerland AG 2020

\begin{abstract}
We report on the influence of chamber design on the gas sensing performance of a graphene field-effect-transistor (GFET). A conventional chamber $(\mathrm{V}=400 \mathrm{ml}$ ) and a cap chamber $(\mathrm{V}=1 \mathrm{ml})$, were used to perform dynamic measurements on a GFET. To gain a-priori knowledge on the gas flow in the chambers, Naiver-Stokes and convection-diffusion equations were numerically-solved using COMSOL Multiphysics. We numerically and experimentally observed two main factors that can affect the GFET performance: (1) the gas flow direction through the chamber and (2) the chamber volume. At 5-min exposure time, at least $200 \%$ higher GFET sensitivity was calculated from the cap chamber, which is expected since the conventional chamber is 400 times larger. Interestingly, even when the conventional chamber is fully saturated (at 90 -min exposure time), the GFET sensitivity in the cap chamber is still better by $28.57 \%$. We attributed this behavior to the swirling vapor flow in the cap chamber brought about by the U-shaped path. This effect causes multiple interaction of $\mathrm{H}_{2} \mathrm{O}$ molecules with the GFET, resulting to higher computed sensitivity. However, at higher relative humidity, the GFET becomes populated, reducing the number of $\mathrm{H}_{2} \mathrm{O}$ molecules that can re-interact with the sensor. In terms of GFET transient characteristics, a $154 \%$ and $86.9 \%$ faster response and recovery, respectively, were observed in the cap-design. This was due to its smaller volume that minimized poorly purged region in the chamber. But if the chambers have the same volumes, we may infer a faster GFET response and recovery from the conventional chamber where the gas flow is unperturbed. These results could contribute in designing a time efficient and cost-effective gas sensing system.
\end{abstract}

Keywords Gas sensor · Chamber · COMSOL MultiPhysics · Graphene · Field effect transistor

\section{Introduction}

Research on gas sensing technology had been widely explored for the past decades. One of the major parameters in the development of gas sensing applications is the design of the measurement system. Normally, sensors are tested using the following techniques [1, 2]: (1) static system and (2) dynamic system. The former typically consists of an airtight chamber, with vacuum levels around $1.333 \mathrm{x}$ $10^{-5} \mathrm{mbar}$, that has a gas inlet for injection of the test gas
[3-8]; while the latter uses continuous gas flow through the chamber [9-14].

Materials modifications [15-19], sensors design [20-24], and testing schemes [25-29], were used to improve the performance of gas sensor devices; but only a few [30-32] reports on its dependence with the chamber design. This factor is important to efficiently investigate the characteristics of the sensor being tested. With an optimized design, turbulence and back flow are minimized, resulting to a more uniform gas distribution inside the chamber. In

$\triangle$ Lorenzo Lopez Jr. , Iplopez@msep.upd.edu.ph | ${ }^{1}$ Materials Science and Engineering Program, University of the Philippine Diliman, 1101 Quezon City, Philippines. ${ }^{2}$ National Institute of Physics, University of the Philippines Diliman, 1101 Quezon City, Philippines. ${ }^{3}$ Berkeley Sensor and Actuator Center \& Mechanical Engineering Department, University of California Berkeley, Berkeley City, CA 94720, USA. 
effect, sensor characteristics (i.e. sensitivity, response, and recovery) can be accurately investigated.

Recently, Niyat et al. [33] experimentally and numerically studied the gas sensing performance of tin oxide/ reduced graphene oxide $\left(\mathrm{SnO}_{2} / \mathrm{rGO}\right)$ inside a cylindrical chamber. They concluded that the pressure distribution in such a system is higher on the edges than at the center. Moreover, Endres et al. [31], compared the gas sensor array sensing performance in linear and circular measurement chambers. They experimentally showed that the former provides a shorter purging time ( $25 \mathrm{~s}$ for $500 \mathrm{sccm}$ ), while the latter allows for a more uniform gas flow.

In this work, we compared the performance of a graphene field-effect-transistor (GFET) in conventional ( $\mathrm{V}=$ $400 \mathrm{ml}$ ) and cap ( $\mathrm{V}=1 \mathrm{ml}$ ) chambers in a dynamic system. Our experimental results, along with simulations using Naiver-Stokes and convection-diffusion equations, show that (i) the gas flow direction in the chamber significantly affects the GFET sensitivity; and (ii) a smaller chamber volume minimizes poorly purged regions resulting to a faster GFET response and recovery. This study may be useful in optimizing a chamber design to efficiently investigate the device under test.

\section{Experimental details}

\subsection{GFET fabrication}

The GFET was fabricated via a shadow-masking technique. Three laser-cut masks were used to form the patterns of the metal electrodes and the graphene channel. First, electrodes ( $50 \mathrm{~nm} \mathrm{Au} / 25 \mathrm{~nm} \mathrm{Pd}$ ) were deposited on the graphene substrate (Graphenea [34]: $10 \mathrm{~mm} \times 10 \mathrm{~mm}$ of monolayer graphene on $\mathrm{SiO}_{2} / \mathrm{Si}$ ) using electron beam deposition (schematic diagram shown in Fig. 1). Then the graphene channel was defined via a two-step etching procedure using $\mathrm{O}_{2}$ plasma. Lastly, the metal electrodes were connected to the ceramic packaging using a wire bonder.

\subsection{Graphene characterization and GFET testing}

To investigate the quality of the graphene after the fabrication process, Raman spectroscopy and scanning electron microscopy (SEM) were performed. The Raman signal of graphene was obtained using a $514 \mathrm{~nm}$ wavelength excitation source, with a power of $5 \mathrm{~mW}$ and a beam size of $\sim$ $1 \mu \mathrm{m}$. The micrograph of the device was captured using a Schottky field emission SEM (ZEISS-SEM).

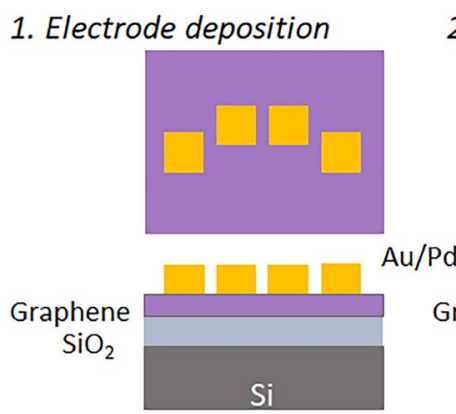

2. Graphene etching (I)

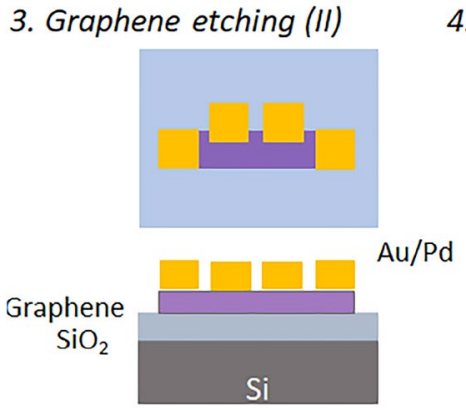

4. Wire bonding

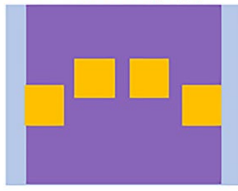

Graphene $\mathrm{SiO}_{2}$

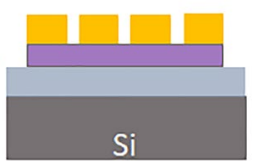

S

Fig. 1 Fabrication process of GFET. (1) Metal electrodes deposition of $\mathrm{Au} / \mathrm{Pd}(50 \mathrm{~nm} / 25 \mathrm{~nm}$ ) using electron beam deposition, (2)-(3) Graphene etching, (4) wire bonding of GFET on test chip

Prior to the gas sensing experiment, the gate voltage $\left(V_{g}\right)$ was modulated, to ensure that the GFET is working properly. The $\mathrm{V}_{g}$ and source-drain current $\left(\mathrm{I}_{s d}\right)$ were supplied using a high accuracy DC power supply, while a 7.5-digit precision digital multimeter was used to measure the potential drop across $\mathrm{V}_{a}$ and $\mathrm{V}_{b}$ (Fig. 2a). $1 \mu \mathrm{A}$ and $-3 \mathrm{~V}$ were used as the $\mathrm{I}_{s d}$ and $\mathrm{V}_{g}$, respectively, to comply with the condition for wearable devices which requires ultra-low power consumption.

To properly control the gas flow through the chamber, mass flow controllers (Omega FMA3706) were incorporated to the gas sensing system. Target vapor concentrations of $20 \%, 35 \%$ and $50 \%$ were produced by bubbling $\mathrm{H}_{2} \mathrm{O}$ using high purity (99.98\%) $\mathrm{N}_{2}$ gas and was then fed through the chamber. The total flow rate of the injected gas was set to $200 \mathrm{sccm}$.

We used two chamber designs: (i) conventional $(\mathrm{V}=$ $400 \mathrm{ml}$ ) and (ii) cap ( $\mathrm{V}=1 \mathrm{ml}$ ) chambers. Our cap chamber has a U-shape path for the gas flow direction, and linear for the conventional chamber. The cap chamber is made from delrin installed with BNC cable for electrical connections, while the conventional chamber is composed of stainless steel equipped with screw terminal 

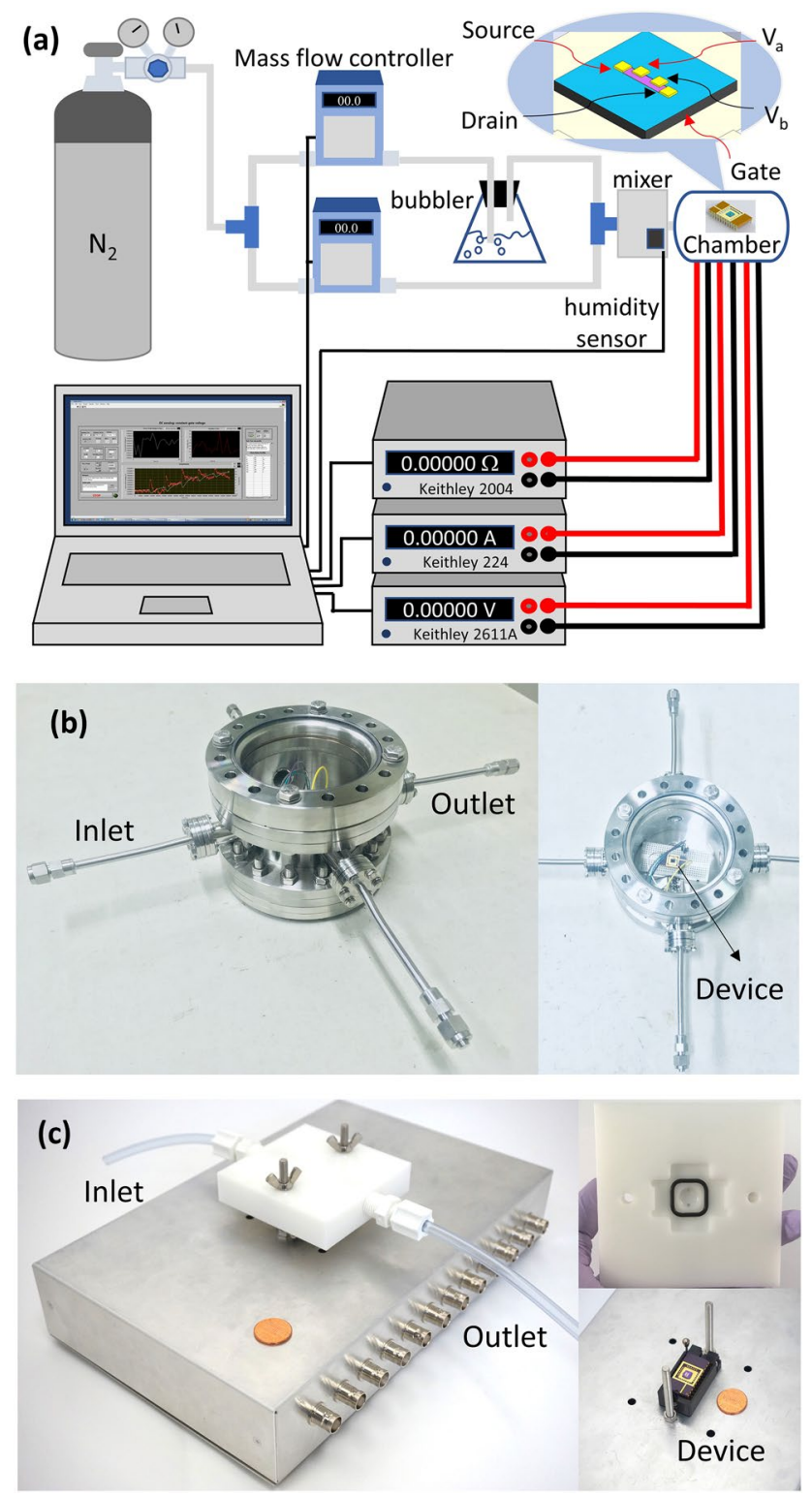

Fig. 2 a Schematic diagram of gas sensor testing system showing the gas line and electrical connections. Actual image of $\mathbf{b}$ conventional chamber and c cap chamber with the mounted GFET device

wires, as shown in Fig. $2 b$ and c, respectively. For the cap chamber, the sensor is mounted on a fixed integrated circuit socket, and on a breadboard for the conventional chamber. A commercial humidity sensor ( $\mathrm{HIH}-4000-001)$ was also integrated to the system to monitor the relative humidity (R.H.). The entire gas sensing system was automated for ease of data collection.

\section{Results and discussion}

\subsection{Properties of graphene channel}

The effective area of graphene channel was measured to be $2.4 \mathrm{~mm}$ by $0.4 \mathrm{~mm}$, as shown in Fig. 3a. Since shadowmasking technique was utilized to pattern the electrodes, it is expected that the metal will diffuse during the deposition process which appears as the dark regions around the electrodes.

Figure $3 \mathrm{~b}$ shows the Raman spectrum of monolayer graphene before and after fabrication process. The prominent peaks at $1340.1 \mathrm{~cm}^{-1}, 1592.9 \mathrm{~cm}^{-1}$, and $2680.3 \mathrm{~cm}^{-1}$ correspond to the D-peak, G-peak and 2D-peak respectively [35]. The enhancement of the D-peak signifies that defects were introduced to graphene after fabrication process [36]. The intensity ratio of the 2D-peak and the 2G-peak $\left(\mathrm{I}_{2 D} / \mathrm{I}_{G}\right)$ of graphene before and after fabrication are 1.4 and 1.0, respectively; signifying the presence of a monolayer graphene even after the fabrication process [35]. Due to instrument limitations, the neutrality point from the $I_{s d}-V_{g}$ curve (Fig. $3 c$ ) cannot be observed. A GFET using an intrinsic graphene is expected to have a Dirac point close to $0 \mathrm{~V}_{g}$ [37]. But since the substrate that we used is $\mathrm{SiO}_{2}$, when exposed to ambient environment, it induces an involuntary p-type doping [38, 39]. This shifts the Dirac point to the positive side in terms of $\mathrm{V}_{g}$, in this case, above $+80 \mathrm{~V}$, beyond our sweeping voltage range. Nonetheless, the fabricated GFET was successfully modulated from - 80 $\mathrm{V}$ to $+80 \mathrm{~V}$, indicating that the device is working properly.

\subsection{COMSOL multiphysics simulation of gas flow}

The gas flow through the chamber is modeled using laminar flow by solving Navier-Stokes equation [40] which is given by,

$\rho \frac{D \bar{U}}{D t}=-\nabla P+\rho g+\eta \nabla^{2} U$

where $\rho, U, P, g$, and $\eta$ are the mass density, velocity field, gas pressure, acceleration due to gravity, and viscosity, respectively. The expression on the left side corresponds to the inertial forces which is the superposition of pressure forces (first term), buoyancy forces (second term), and viscous forces (third term). Equation 1 can also be expressed into Cartesian coordinates as follows: 
Fig. 3 a SEM image of the GFET showing the graphene channel and patterned electrodes; b Raman signal shows the presence of graphene even after the fabrication process; and c Gate voltage modulation of GFET signifying that the device is working properly
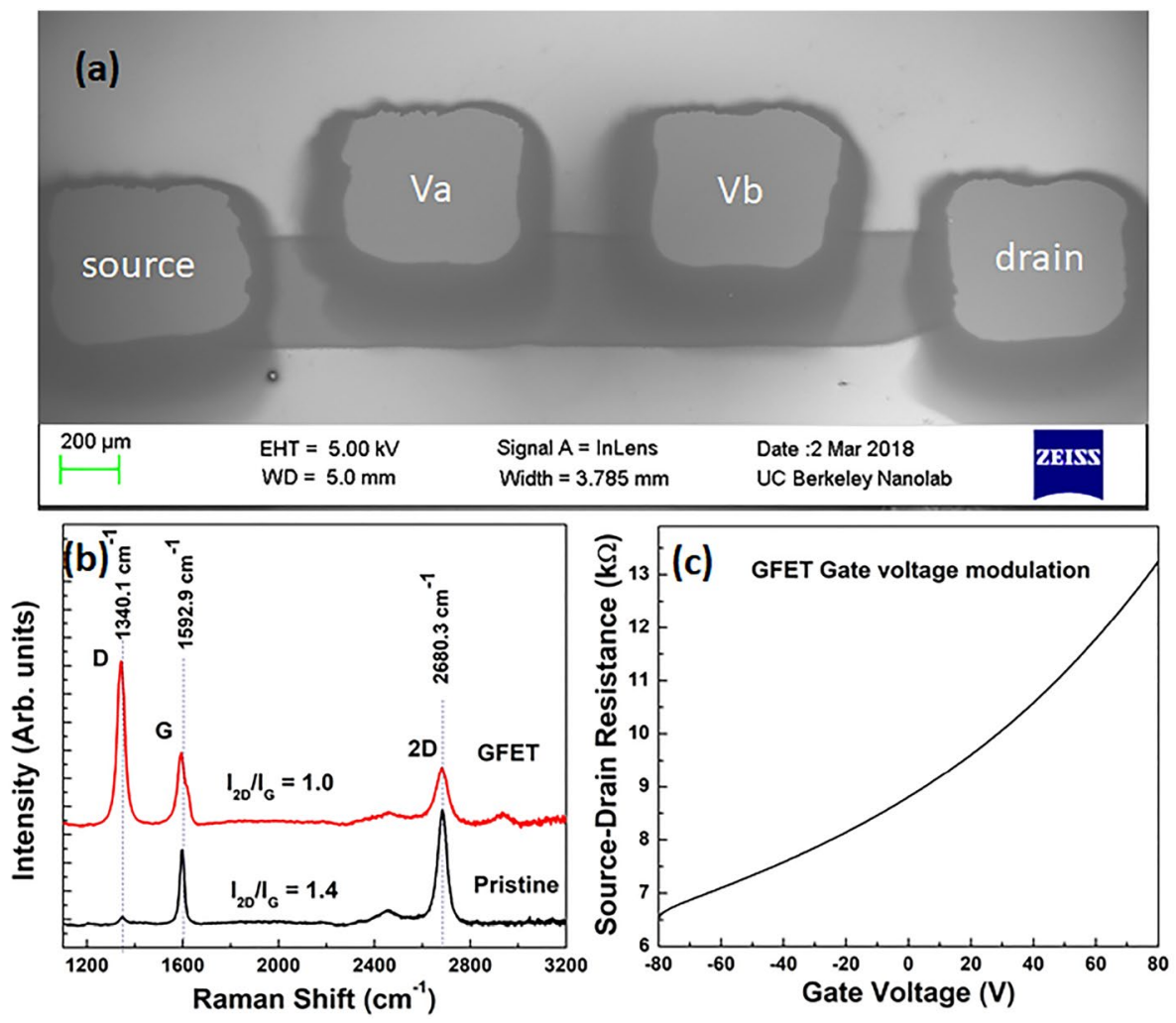

$$
\begin{aligned}
X & : \rho\left(\frac{\partial U}{\partial t}+U \frac{\partial U}{\partial x}+V \frac{\partial U}{\partial y}+W \frac{\partial U}{\partial z}\right) \\
& =-\frac{\partial P}{\partial x}+\rho g_{x}+\mu\left(\frac{\partial^{2} U}{\partial x^{2}}+\frac{\partial^{2} U}{\partial y^{2}}+\frac{\partial^{2} U}{\partial z^{2}}\right) \\
Y & : \rho\left(\frac{\partial V}{\partial t}+U \frac{\partial V}{\partial x}+V \frac{\partial V}{\partial y}+W \frac{\partial V}{\partial z}\right) \\
& =-\frac{\partial P}{\partial y}+\rho g_{y}+\mu\left(\frac{\partial^{2} V}{\partial x^{2}}+\frac{\partial^{2} V}{\partial y^{2}}+\frac{\partial^{2} V}{\partial z^{2}}\right) \\
Z & : \rho\left(\frac{\partial W}{\partial t}+U \frac{\partial W}{\partial x}+V \frac{\partial W}{\partial y}+W \frac{\partial W}{\partial z}\right) \\
& =-\frac{\partial P}{\partial z}+\rho g_{z}+\mu\left(\frac{\partial^{2} W}{\partial x^{2}}+\frac{\partial^{2} W}{\partial y^{2}}+\frac{\partial^{2} W}{\partial z^{2}}\right)
\end{aligned}
$$

To obtain the field velocity of the gas flowing through the chamber, Navier-Stokes equation must be simultaneously solved with the continuity equation, which is given by $\frac{\partial \rho}{\partial t}+\nabla \cdot(\rho \mu)=0$

For incompressible gas flow, this equation yields:

$\nabla \cdot \mu=0$

No-slip and adiabatic boundary conditions were set all over the chamber except for the inlet and outlet.

The parameters for the dimensions of the chambers were initially defined to easily generate their geometry, wherein conventional and U-shape chambers have 400 $\mathrm{ml}$ and $1 \mathrm{ml}$ volume, respectively. The meshing was built using a free tetrahedral. A time dependent study, with time profile ranging from $1 \mathrm{~s}$ to $3000 \mathrm{~s}$, was implemented to generate the velocity profile of $\mathrm{H}_{2} \mathrm{O}$ vapor through the chambers, and the $\mathrm{H}_{2} \mathrm{O}$ concentration profile at the device location.

The velocity profile of gas through the conventional and cap chambers are depicted in Fig. 4. The inlet flow rate was set to $200 \mathrm{sccm}$. As expected, the gas flow follows the linear path and U-path directions for the conventional and cap chambers, respectively. Due to the 
Fig. 4 Laminar flow of gas through the a conventional chamber and $\mathbf{b}$ cap chamber showing the $x-z$ plane coordinate and device location. A linear gas flow direction is observed from the conventional chamber, while a U-path direction for the cap chamber. The gas velocity magnitude in cap chamber is 100 times faster than that in the conventional chamber, which is due to dimension differences
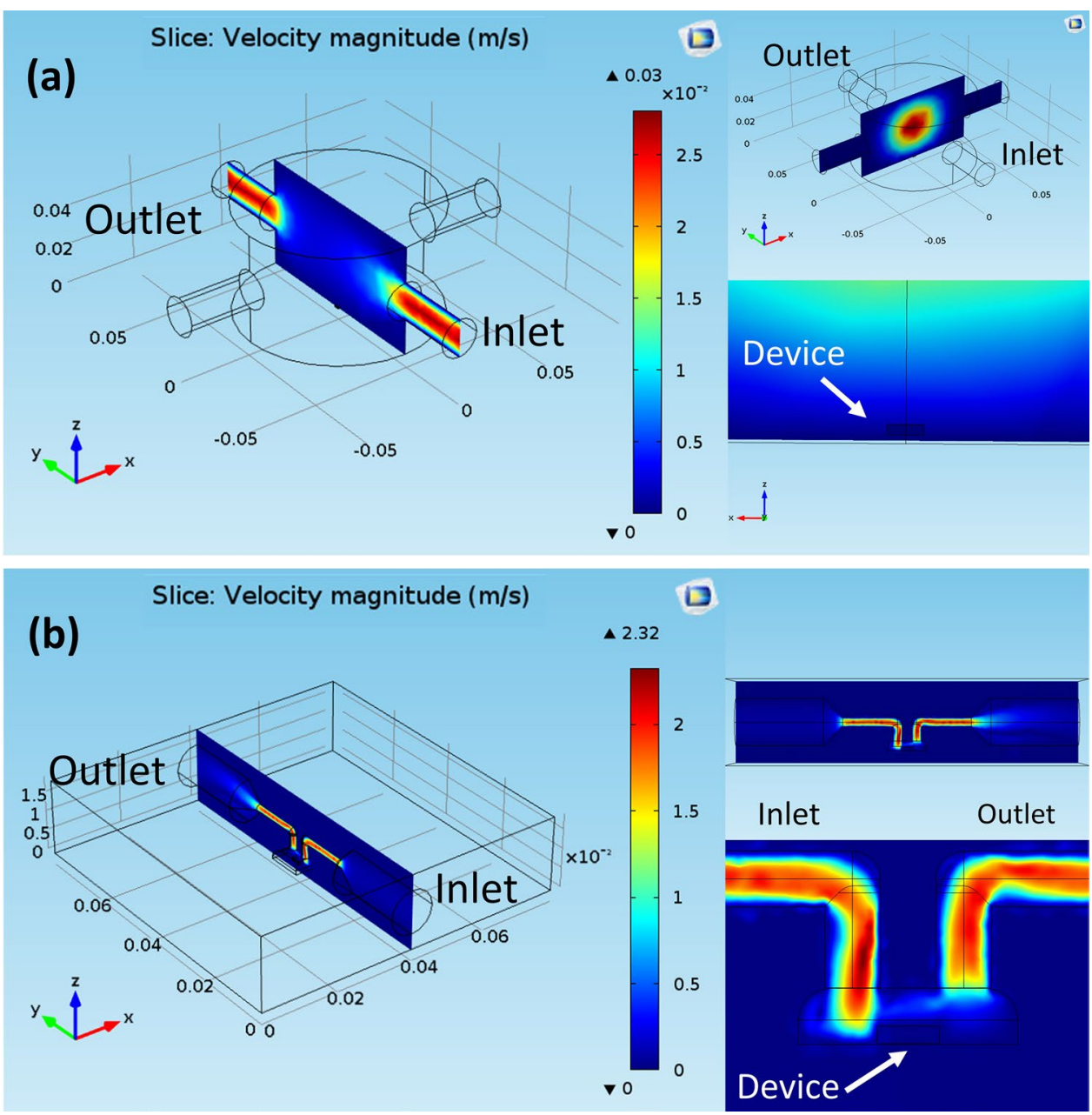

smaller volume of the cap chamber, the velocity magnitude of gas through it is 100 times faster. The gas velocity distribution in the conventional chamber significantly changes depending on the location of the device, due to its large volume. In our simulation, we positioned it at the center, to accurately compare the gas flow in both chambers.

Furthermore, convection-diffusion equation [41] was used to model the gas transport through the chamber, which is given by,

$\frac{\partial c_{i}}{\partial t}+\nabla \cdot\left(-D_{i} \nabla c_{i}+c_{i} u\right)-R_{i}=0$

where $c, D, u$, and $R$ are the concentration, diffusivity, velocity, and production or consumption term, respectively.

The cap chamber reached the target set point at 35\% $\mathrm{H}_{2} \mathrm{O}$ vapor concentration, for an exposure time $\left(\mathrm{t}_{\text {exp }}\right)$ of 5 mins. At this $t_{\text {exp }}$, the conventional chamber reach only $12 \% \mathrm{H}_{2} \mathrm{O}$ vapor concentration (shown in Fig. 5). This is expected since it has a larger volume. Based from our simulation, the time required for the conventional chamber to reach the target set point is 90 mins. At saturation levels, we can assume that the number of $\mathrm{H}_{2} \mathrm{O}$ molecules interacting with the device in both chambers are approximately equal. We can therefore abate the effect of volume differences and effectively compare gas flow characteristics. These simulation results were also investigated experimentally in the following section.

\subsection{Device testing of GFET}

Figure 6 shows the GFET response to different $\mathrm{H}_{2} \mathrm{O}$ vapor concentrations, using the conventional and cap chambers. The inset shows the R.H. measured by the commercial sensor. For the cap chamber, the target set points of 


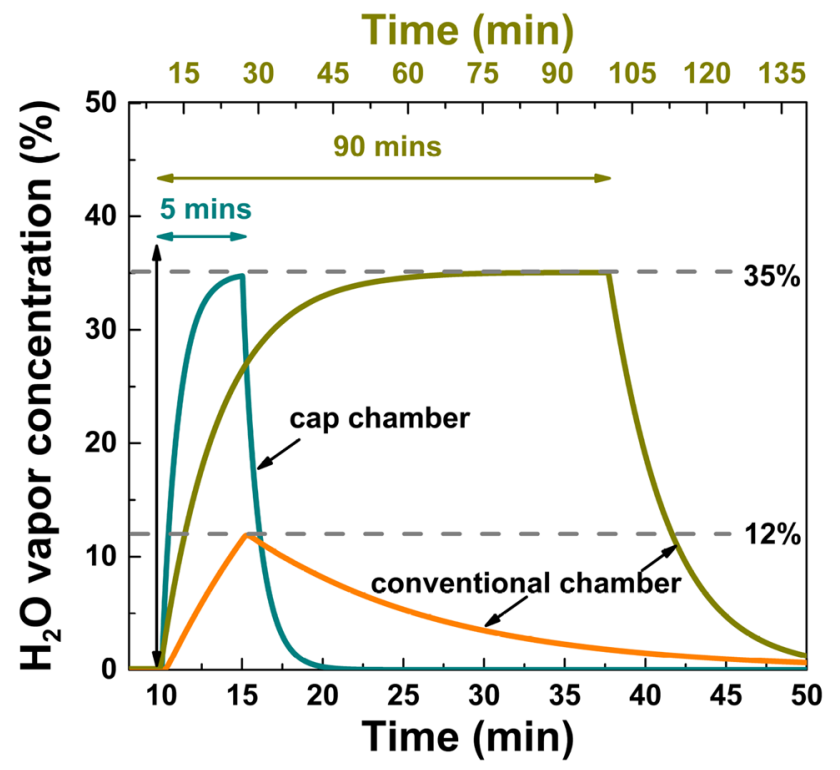

Fig. 5 The gas transport of $\mathrm{H}_{2} \mathrm{O}$ vapor through the conventional chamber with $t_{\exp }=5$ mins (orange line) and $t_{\exp }=90$ mins (green line), and cap chamber with $t_{\text {exp }}=5$ mins (cyan line). The cap chamber reach the target set point at $35 \%$ at $\mathrm{t}_{\text {exp }}=5$ mins, while the conventional chamber requires $t_{\exp }=90$ mins. Double $x$-axis was used for comparison purposes

$20 \%, 35 \%$ and $50 \%$ R.H. were reached at $\mathrm{t}_{\text {exp }}=5$ mins. This agrees well with the simulation results presented in the previous section. Using the same exposure time, the sensor response in the conventional chamber barely reach half of the target set points. Additionally, after the sensor's recovery, a slight increase in its response can be noticed. This can be attributed to the non-uniformity of $\mathrm{H}_{2} \mathrm{O}$ vapor distribution, which may be an effect of turbulence and back flow inside the large-volume conventional chamber. This effect was minimized when the GFET is exposed for 90 mins, which indicates that the chamber is fully saturated and the $\mathrm{H}_{2} \mathrm{O}$ molecules interacting with the GFET is more uniform.

The GFET sensitivity (S) can be written as,

$S(\%)=\left|\frac{\Delta R}{R_{0}}\right| \times 100$

where $\Delta R, R_{0}$ are the change of GFET resistance, and the GFET resistance in the carrier gas, respectively.

The calculated sensitivities of the GFET in conventional and cap chambers for different R.H. levels, are tabulated in
Table 1. At $\mathrm{t}_{\text {exp }}=5$ mins, we can observe at least $200 \%$ GFET sensitivity improvement from the cap chamber. This is expected since the conventional chamber volume is 400 times larger than that of the cap chamber. But even when the conventional chamber is fully saturated (at $t_{\text {exp }}=90$ mins), the GFET sensitivity is still better by $28.57 \%$ (for R.H. $=20 \%$ ). At $50 \%$, however, the GFET sensitivities in the two chambers become comparable. The sensitivity ratios $\left(\left|\frac{\Delta R}{R_{0}}\right|\right.$ cap $\left./\left|\frac{\Delta R}{R_{0}}\right|_{\text {conventional }}\right)$ are shown in Fig. 7. Generally, at higher R.H., the ratio decreases and approaches the simulation values. The ratios fit better with the simulation when both the chambers are fully saturated (Fig. 7b).

The differences in the sensitivity is attributed to the different gas flow directions for each chamber (illustrated in Fig. 8). The U-path in the cap design may have induced a swirling vapor flow in the chamber, introducing multiple interaction of $\mathrm{H}_{2} \mathrm{O}$ molecules with the device, which resulted to the higher calculated sensitivity at lower R.H. (20\% and 35\%). However, at higher R.H. (50\%), the GFET becomes more populated, reducing the number of $\mathrm{H}_{2} \mathrm{O}$ molecules that can re-interact with the device in the cap chamber. This means that, at higher R.H., the number of $\mathrm{H}_{2} \mathrm{O}$ molecules interacting with the GFET in both the chambers will be comparable, resulting to approximately equal sensitivities. The swirling vapor effect was also observed by Zhang et al. [42], but for a different application, i.e. in a U-shaped chemical vapor deposition chamber.

The response and recovery time of the GFET were obtained using Eq. 9 and Eq. 10 respectively.

$t_{\text {res }}=t_{90}$

$t_{\text {rec }}=t_{10}-t_{\max }$

where $t_{\max }, t_{90}$ and $t_{10}$ are the time at maximum sensor response, time at the sensor response is $90 \%$ of the maximum, and time at the sensor response is $10 \%$ of the maximum, respectively.

Figure 9 shows the GFET response and recovery times in the conventional and cap chambers. It can be noted that at higher R.H., the GFET response does not vary very much, and its recovery is slower. The fast response and slow recovery in GFET can be explained by the interaction of $\mathrm{H}_{2} \mathrm{O}$ molecules with the graphene $/ \mathrm{SiO}_{2}$ layers.

According to Smith et al. [43], this humidity sensing effect in graphene/ $\mathrm{SiO}_{2}$ layers is attributed to the interaction of graphene layer and impurity bands in $\mathrm{SiO}_{2}$, with the $\mathrm{H}_{2} \mathrm{O}$ electrostatic dipole moment. In effect, a 

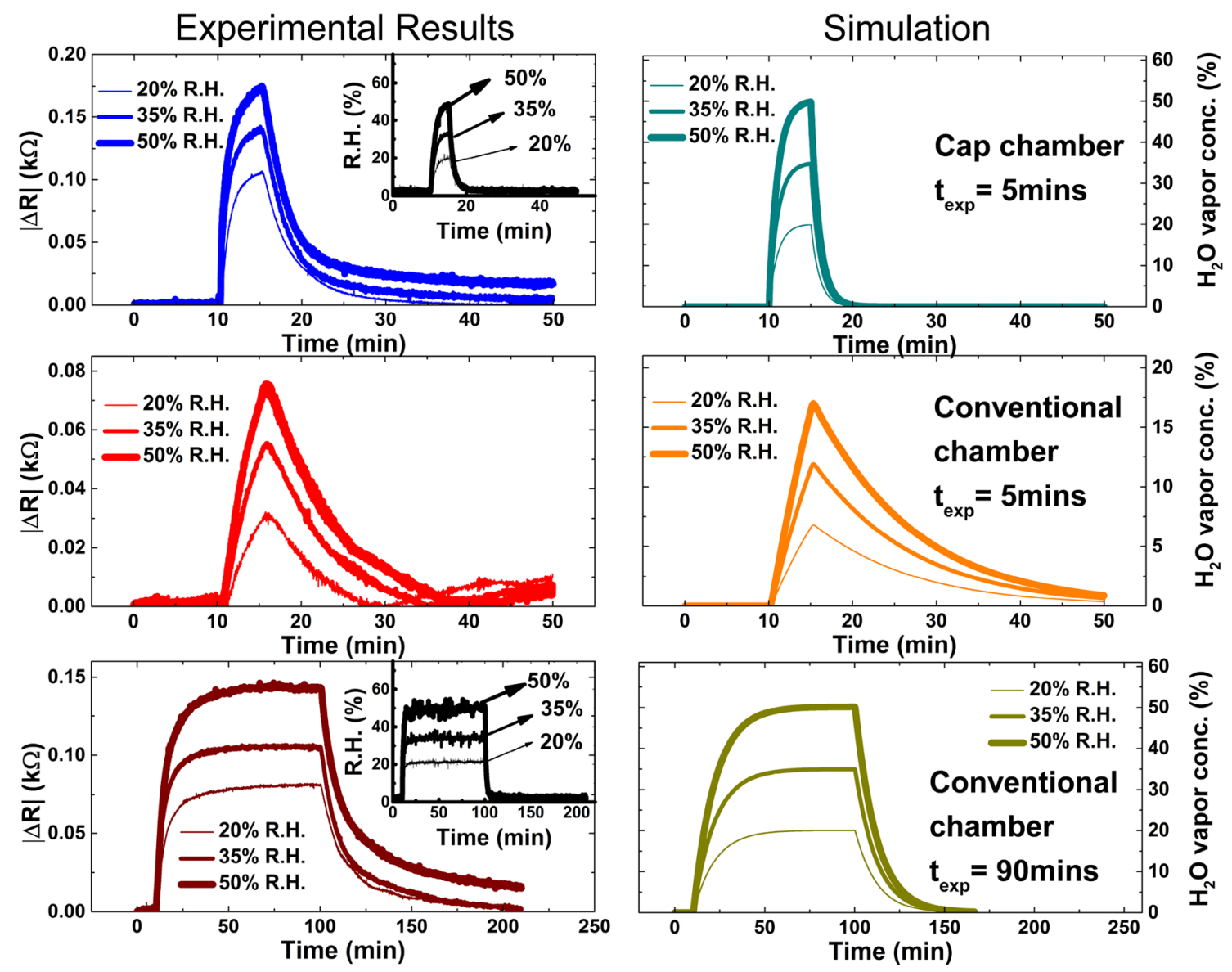

Fig. 6 The GFET response in a cap chamber and a conventional chamber (left) with its corresponding simulations (right). The GFET was exposed for 5 mins (top) in the cap chamber, and 5 mins (middle) and 90 mins (bottom) in the conventional chambers using $20 \%, 35 \%$ and $50 \% \mathrm{H}_{2} \mathrm{O}$ vapor concentrations. Both the experi-

mental and simulation results show that the GFET response reach the target set point in the cap chamber at $t_{\text {exp }}=5$ mins, while in the conventional chamber $t_{\text {exp }}=90$ mins is required. The relative humidity was monitored and shown in the inset

Table 1 Summary of the calculated GFET sensitivities in the cap and conventional chambers under the influence of different R.H. levels. At lower R.H. level, the GFET exhibits better sensitivity in cap chamber compared to that of in a conventional chamber

\begin{tabular}{|c|c|c|c|}
\hline \multirow[t]{2}{*}{ R.H. (\%) } & \multicolumn{3}{|l|}{ GFET sensitivity } \\
\hline & $\begin{array}{l}\text { Cap chamber } \\
\text { (\%) }\end{array}$ & $\begin{array}{l}\text { Conventional } \\
\text { chamber }^{\mathrm{a}}(\%)(\%)\end{array}$ & $\begin{array}{l}\text { Conventional } \\
\left.\text { chamber }{ }^{\text {b }} \%\right) \\
(\%)\end{array}$ \\
\hline 20 & 1.2 & 0.4 & 0.9 \\
\hline 35 & 1.5 & 0.6 & 1.2 \\
\hline 50 & 2.0 & 0.9 & 1.9 \\
\hline
\end{tabular}

${ }^{a} t_{\text {exp }}=5$ mins

$b_{t_{\text {exp }}}=90$ mins

graphene $/ \mathrm{SiO}_{2}$-based sensor will have a faster response time and slower recovery time. Due to the smaller volume of the cap chamber, a faster GFET response (by at least $154 \%$ ) and recovery (by at least $86.9 \%$ ) are expected, as shown in Table 2. A smaller chamber volume minimizes the poorly purged region. But when volumes are equal, we may expect a faster GFET response and recovery in the conventional design, due to the unperturbed gas flow in the chamber's linear path direction.

On a related note, Annanouch et al. [44] also demonstrated the effect of chamber design, but for a $\mathrm{SnO}_{2}$ chemiresistor. Their results showed that a boat-shaped chamber $(\mathrm{V}=2.35 \mathrm{ml}$ ) have faster response and recovery 

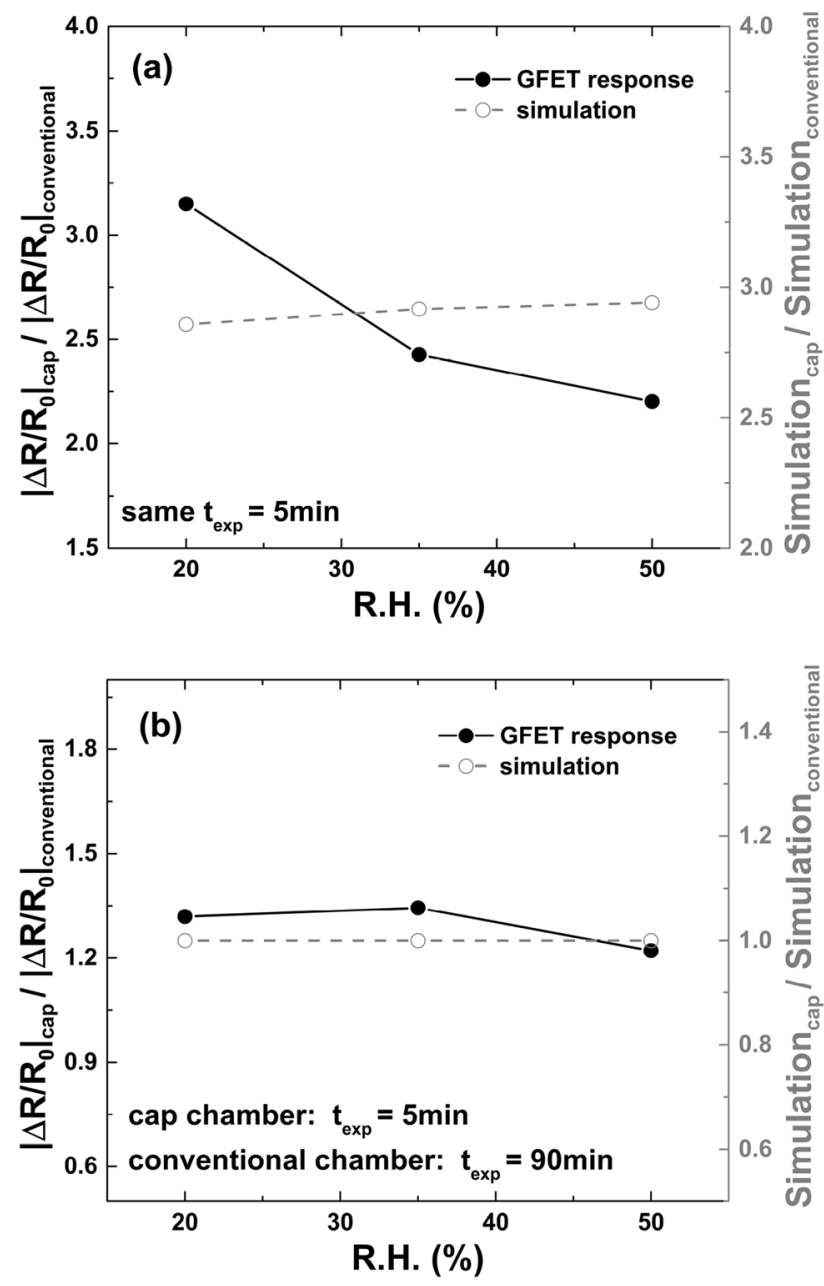

Fig. 7 The GFET sensitivity ratio in the conventional and cap chambers for the $\mathbf{a}$ same exposure time, and for $\mathbf{b}$ different exposure times along with its corresponding simulations. Generally, At higher R.H., the $\left|\frac{\Delta R}{R_{0}}\right|_{\text {cap }} /\left|\frac{\Delta R}{R_{0}}\right|_{\text {conventional }}$ decreases and approaches the simulation values times compared to a cross-shaped chamber ( $\mathrm{V}=300 \mathrm{ml})$. They attributed this to the enhanced gas concentration homogeneity and reduced dead volume in the boatshaped chamber. But in our case, aside from the effect of volume, we surmise that the improvement in the detected target gas is also based on the re-interaction of the gas molecules with the sensor, brought about by the U-shaped path in the cap chamber.

\section{Conclusion}

We successfully investigated the effect of chamber design on the gas sensing performance of a GFET, via comparing GFET performances in conventional $(V=400$ $\mathrm{ml})$ and cap $(\mathrm{V}=1 \mathrm{ml})$ chambers. At saturation levels (abating volume differences), a higher GFET sensitivity was observed in the cap chamber for lower R.H. levels (20\% and $35 \%)$. This was attributed to the swirling vapor effect in the U-path direction of the chamber, causing multiple interaction of $\mathrm{H}_{2} \mathrm{O}$ molecules to the GFET. At high concentration (50\% R.H.), however, this effect is minimized, resulting to comparable GFET sensitivities between the chambers. Furthermore, faster GFET response and recovery times were observed in the cap chamber. The smaller volume of the cap-design minimizes the poorly purged region in the chamber. But if the chambers have the same volumes, faster GFET response and recovery may be expected from the conventional chamber, where the gas flow direction is unperturbed. This study can contribute not only on the optimization of gas chamber design for efficient device testing; but
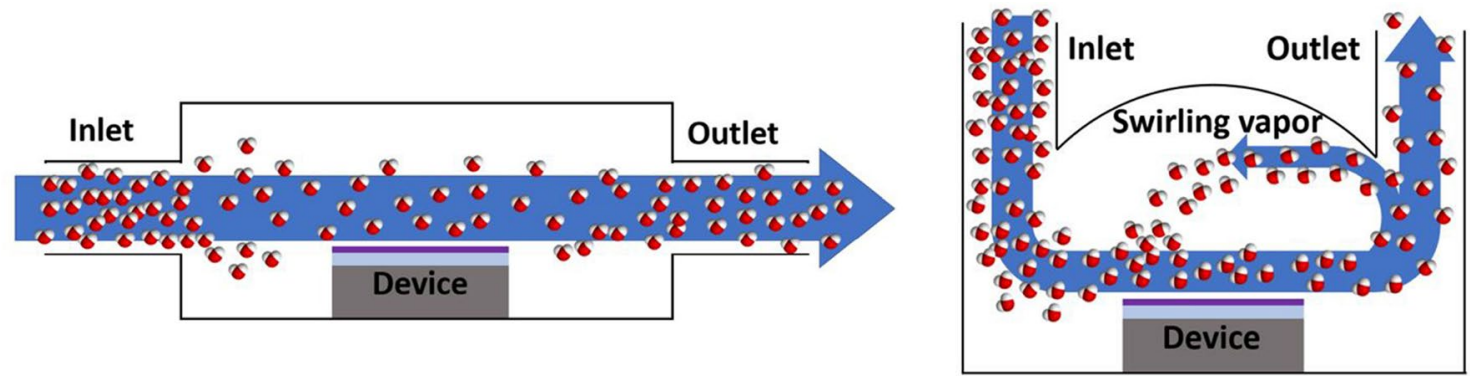

Fig. 8 Illustration of gas flow through the conventional chamber (top) and the cap chamber (bottom). The gas flow in the cap chamber follows a U-path direction that resulted to a swirling vapor, while a linear-path in the conventional chamber, wherein the gas flow is unperturbed. In effect, a better sensitivity is observed in the cap chamber 

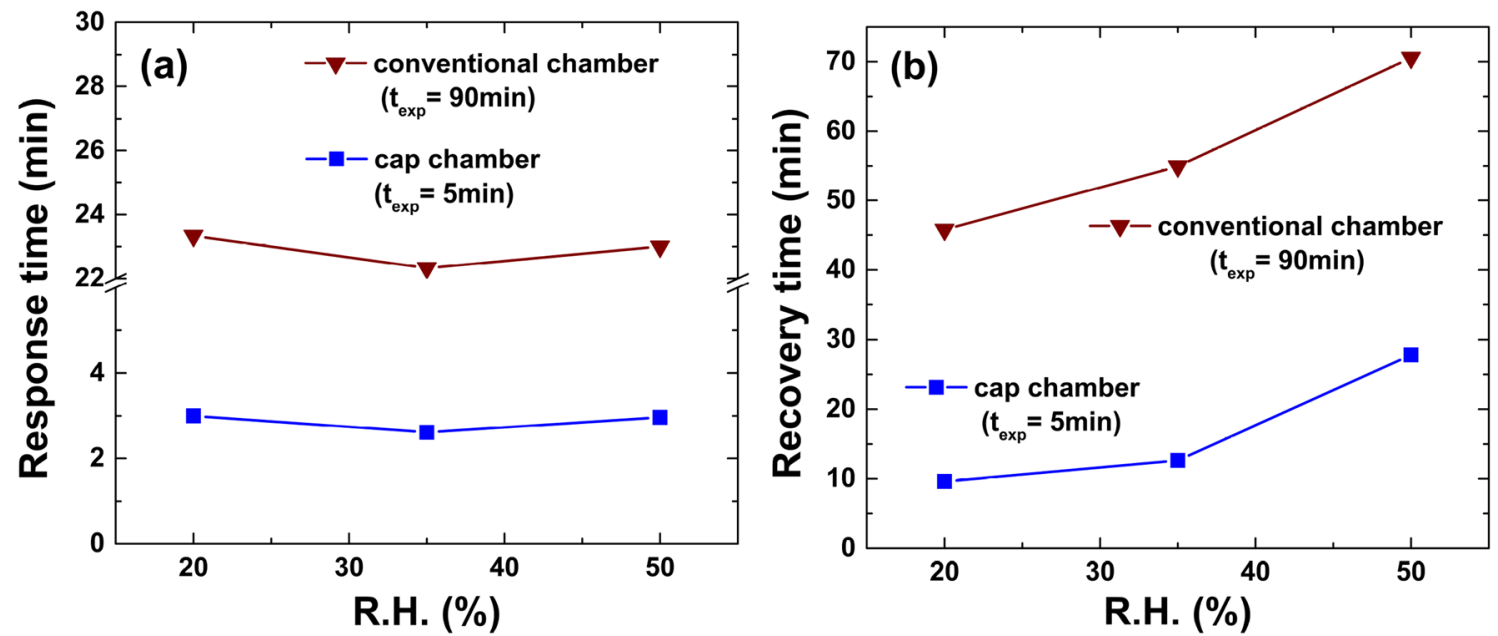

Fig. 9 The $\mathbf{a}$ response time and $\mathbf{b}$ recovery time of GFET in the conventional and cap chambers under the influence of different humidity levels. The GFET transient characteristic is better in the cap chamber due to its smaller volume compared to the conventional chamber

Table 2 Summary of the calculated GFET response and recovery in the cap and conventional chambers under the influence of different R.H. levels. The percent difference (\% diff.) for the $t_{r e s}$ and $t_{r e c} u s i n g$ the cap and conventional chambers were calculated

\begin{tabular}{|c|c|c|c|c|c|c|}
\hline \multirow[t]{2}{*}{ R.H. (\%) } & \multicolumn{3}{|l|}{$t_{\text {res }}$} & \multicolumn{3}{|l|}{$t_{r e c}$} \\
\hline & $\mathrm{Cap}^{\mathrm{a}}$ & Con. ${ }^{b}$ & $\%$ diff. & $\mathrm{Cap}^{\mathrm{a}}$ & Con. Con. ${ }^{b}$ & $\%$ diff. \\
\hline 20 & 3.0 & 23.3 & 154.4 & 9.6 & 45.7 & 130.6 \\
\hline 35 & 2.6 & 22.3 & 158.0 & 12.6 & 54.9 & 125.2 \\
\hline 50 & 2.9 & 23.0 & 154.3 & 27.8 & 70.6 & 86.9 \\
\hline
\end{tabular}

$\mathrm{a}_{\mathrm{t}_{\text {exp }}}=5 \mathrm{mins}$

$b_{t_{\text {exp }}}=90$ mins

also on possible applications in microfluidics and drug delivery, wherein efficient fluid transport is required.

Acknowledgements This work was supported by the PhilippineCalifornia Advanced Research Institute (PCARI) under the project of "Chemical and Environment - Portable Sensor Technologies (CEPoST)" with Grant Number IIID-2016-010, and the Berkeley Sensor and Actuator Center (BSAC). The device was fabricated at the University of California Berkeley (UCB) Marvell Nanofabrication Laboratory (Nanolab).

\section{Compliance with ethical standards}

Conflict of interest The authors declare that they have no conflict of interest.

\section{References}

1. Mirzaei A, Kim SS, Kim HW (2018) Resistance-based $\mathrm{H}_{2} \mathrm{~S}$ gas sensors using metal oxide nanostructures: A review of recent advances. J Hazard Mater 357:314-331. https://doi. org/10.1016/j.jhazmat.2018.06.015
2. Karunagaran $B$, Uthirakumar $P$, Chung SJ, Velumani S, Suh EK (2007) $\mathrm{TiO}_{2}$ thin film gas sensor for monitoring ammonia. Mater Charact 58:680-684. https://doi.org/10.1016/j.match ar.2006.11.007

3. Li S, Cheng M, Liu G, Zhao L, Zhang B, Gao Y, Lu H, Wang H, Zhao J, Liu F, Yan X (2018) High-response and low-temperature nitrogen dioxide gas sensor based on gold-loaded mesoporous indium trioxide. J Colloid Interface Sci 524:368-378. https://doi. org/10.1016/j.jcis.2018.04.033

4. Patil UV, Ramgir NS, Karmakar N, Bhogale A, Debnath AK, Aswal DK, Gupta SK, Kothari DC (2015) Room temperature ammonia sensor based on copper nanoparticle intercalated polyaniline nanocomposite thin films. Appl Surf Sci 339:69-74. https://doi. org/10.1016/j.apsusc.2015.02.164

5. Kaviyarasu K, Mola GT, Oseni SO, Kanimozhi K, Magdalane CM, Kennedy J, Maaza M (2019) ZnO doped single wall carbon nanotube as an active medium for gas sensor and solar absorber. Mater Sci Mater Electron 30:147-158. https://doi.org/10.1007/ s10854-018-0276-6

6. Liu F, Yang X, Wang B, Guan Y, Liang X, Sun P, Lu G (2019) High performance mixed potential type acetone sensor based on stabilized zirconia and $\mathrm{NiNb}_{2} \mathrm{O}_{6}$ sensing electrode. Sens Actuators B Chem 229:200-208. https://doi.org/10.1016/j.snb.2016.01.128

7. Li Z (2017) Supersensitive and superselective formaldehyde gas sensor based on NiO nanowires. Vacuum 143:50-53. https://doi. org/10.1016/j.vacuum.2017.05.038 
8. Liang J, Liu J, Li W, Hu M (2016) Preparation and room temperature methane sensing properties of platinum-decorated vanadium oxide films. Mater Res Bull 84:332-339. https://doi. org/10.1016/j.materresbull.2016.08.024

9. Leidinger $M$, Schultealbert $C$, Neu J, Schütze A, Sauerwald T (2017) Characterization and calibration of gas sensor systems at $\mathrm{ppb}$ level-A versatile test gas generation system. Meas Sci Technol 29:015901. https://doi.org/10.1088/1361-6501/aa91da

10. Maziarz W, Pisarkiewicz T (2008) Gas sensors in a dynamic operation mode. Meas Sci Technol 19:055205. https://doi. org/10.1088/0957-0233/19/5/055205

11. Fonollosa J, Sheik S, Huerta R, Marco S (2015) Reservoir computing compensates slow response of chemosensor arrays exposed to fast varying gas concentrations in continuous monitoring. Sens Actuators B Chem 215:618-629. https://doi.org/10.1016/j. snb.2015.03.028

12. Goutham S, Sadasivuni KK, Kumar DS, Rao KV (2018) Flexible ultra-sensitive and resistive $\mathrm{NO}_{2}$ gas sensor based on nanostructured $\mathrm{Zn}(x) \mathrm{Fe}(1--x) 2 \mathrm{O}$. RSC Adv 8:3243-3249. https://doi. org/10.1039/C7RA10478B

13. Perillo PM, Rodríguez DF (2016) Low temperature trimethylamine flexible gas sensor based on $\mathrm{TiO}_{2}$ membrane nanotubes. J Alloy Compd 657:765-769. https://doi.org/10.1016/j.jallc om.2015.10.167

14. Bailly G, Harrabi A, Rossignol J, Stuerga D, Pribetich P (2016) Microwave gas sensing with a microstrip interDigital capacitor: detection of $\mathrm{NH}_{3}$ with $\mathrm{TiO}_{2}$ nanoparticle. Sens Actuators B Chem 236:554-564. https://doi.org/10.1016/j.snb.2016.06.048

15. Ma L, Fan H, Tian H, Fang J, Qian X (2016) The $n-Z n O / n-I n_{2} \mathrm{O}_{3}$ heterojunction formed by a surface-modification and their potential barrier-control in methanal gas sensing. Sens Actuators B Chem 222:508-516. https://doi.org/10.1016/j.snb.2015.08.085

16. Tang $X$, Haddad PA, Mager N, Geng X, Reckinger N, Hermans S, Debliquy M, Raskin JP (2019) Chemically deposited palladium nanoparticles on graphene for hydrogen sensor applications. Sci Reports 9:3653. https://doi.org/10.1038/s41598-019-40257 $-7$

17. Roy S, Dey A, Biswas B, Sarkar S (2018) Investigation of Pt and Pd Modified $\mathrm{WO}_{3}$ and $\mathrm{ZnO}$ based thin film sensors for ethanol sensing. J Mater Eng Perform 27:2635-2642. https://doi.org/10.1007/ s11665-017-3105-9

18. Wu J, Tao K, Guo Y, Li Z, Wang X, Luo Z, Feng S, Du C, Chen D, Miao J, Norford L (2017) A 3D chemically modified graphene hydrogel for fast, highly sensitive, and selective gas sensor. Adv Sci 4:1600319. https://doi.org/10.1002/advs.201600319

19. Roy PK, Haider G, Chou TC, Chen KH, Chen LC, Chen YF, Liang CT (2019) Ultrasensitive gas sensors based on vertical graphene nanowalls/SiC/Si heterostructure. ACS Sens 4:406-412. https:// doi.org/10.1021/acssensors.8b01312

20. Meng F, Guo Z, Huang X (2015) Graphene-based hybrids for chemiresistive gas sensors. TrAC Trends Anal Chem 68:37-47. https://doi.org/10.1016/j.trac.2015.02.008

21. Kim J, Lee M, Jeon S, Kim M, Kim S, Kim K, Bien F, Hong S, Park J (2015) Highly transparent and stretchable field-effect transistor sensors using graphene-nanowire hybrid nanostructures. Adv Mater 27:3292-3297. https://doi.org/10.1002/ adma.201500710

22. Tanakaand H, Kanno Y (2007) Quartz crystal capacitive sensor with inductance-capacitance resonance circuit for vapor sensing. Jpn J Appl Phys 46:7509. https://doi.org/10.1143/ JJAP.46.7509

23. Rahbarpour S, Hosseini-Golgoo SM (2013) Diode type Ag-TiO hydrogen sensors. Sens Actuators B Chem 187:262-266. https ://doi.org/10.1016/j.snb.2012.11.017
24. Gao F, Xuan W, Bermak A, Boussaid F, Tsui CY, Luo J (2019) Dual transduction on a single sensor for gas identification. Sens Actuators B Chem 278:21-27. https://doi.org/10.1016/j. snb.2018.09.029

25. Kulkarni G, Reddy K, Zhong Z, Fan X (2014) Graphene nanoelectronic heterodyne sensor for rapid and sensitive vapour detection. Nat Commun 5:4376. https://doi.org/10.1038/ncomm s5376

26. Liu H, Liu Y, Chu Y, Hayasaka T, Joshi N, Cui Y, Wang X, You Z, Lin L (2018) AC phase sensing of graphene FETs for chemical vapors with fast recovery and minimal baseline drift. Sens Actuators $B$ Chem 263:94-102. https://doi.org/10.1016/j.snb.2018.01.244

27. Shin J, Hong Y, Wu M, Bae J, Kwon H, Park B, Lee J (2018) An accurate and stable humidity sensing characteristic of Si FETtype humidity sensor with $\mathrm{MoS}_{2}$ as a sensing layer by pulse measurement. Sens Actuators B Chem 258:574-579. https:// doi.org/10.1016/j.snb.2017.11.132

28. Hu H, Yang X, Guo X, Khaliji K, Biswas SR, García de Abajo FJ, Low T, Sun Z, Dai Q (2019) Gas identification with graphene plasmons. Nature 10:1-7. https://doi.org/10.1038/s4146 7-019-90079

29. Reddeppa M, Mitta SB, Park BG, Kim SG, Park SH, Kim MD (2019) DNA-CTMA functionalized $\mathrm{GaN}$ surfaces for $\mathrm{NO}_{2}$ gas sensor at room temperature under UV illumination. Org Electron 65:334340. https://doi.org/10.1016/j.orgel.2018.11.038

30. Gándara CL, Fernández-Sanjuán JM, Klaas A, Ramos FM, Cirera A (2012) How to test exhaust gas sensors? influence of gas testing systems and experimental artifacts in exhaust gas sensors characterization. Procedia Eng 47:1105-1108. https://doi. org/10.1016/j.proeng.2012.09.344

31. Endres HE, Jander HD, Göttler W (1995) A test system for gas sensors. Sens Actuators B Chem 23:163-172. https://doi. org/10.1016/0925-4005(94)01272-J

32. Annanouch FE, Bouchet G, Perrier P, Morati N, Reynard-Carette C, Aguir K, Bendahan M (2018) How the chamber design can affect gas sensor responses. Multidiscip Dig Publ Instit Proc. https:// doi.org/10.3390/proceedings2130820

33. Niyat FY, Abadi MHS (2018) COMSOL-based modeling and simulation of $\mathrm{SnO}_{2} / \mathrm{rGO}$ gas sensor for detection of $\mathrm{NO}_{2}$. Sci Rep 8:2149. https://doi.org/10.1038/s41598-018-20501-2

34. Graphenea.https://www.graphenea.com

35. Malard LM, Pimenta MAA, Dresselhaus G, Dresselhaus MS (2009) Raman spectroscopy in graphene. Phys Rep 473:51-87. https:// doi.org/10.1016/j.physrep.2009.02.003

36. Beams R, Cançado LG, Novotny L (2015) Raman characterization of defects and dopants in graphene. J Phys Condens Matter 27:083002. https://doi.org/10.1088/0953-8984/27/8/083002

37. Chen S, Cai W, Chen D, Ren Y, Li X, Zhu Y, Kang J, Ruoff RS (2010) Adsorption/desorption and electrically controlled flipping of ammonia molecules on graphene. New J Phys 12:125011. https ://doi.org/10.1088/1367-2630/12/12/125011

38. Shi Y, Dong X, Chen P, Wang J, Li L (2009) Effective doping of single-layer graphene from underlying $\mathrm{SiO}_{2}$ substrates. Phys Rev B 79:115402. https://doi.org/10.1103/PhysRevB.79.115402

39. Liu H, Liu Y, Zhu D (2011) Chemical doping of graphene. J Mater Chem 21:3335-3345. https://doi.org/10.1039/C0JM02922J

40. Kundu PK, Cohen IM, Dowling DR (2016) Fluid Mechanics. Elsevier, Cambridge, Massachusetts, USA, pp 130-162. https://doi. org/10.1016/b978-0-12-405935-1.00004-6

41. Bird RB, Stewart WE, Lightfoot EN (2007) Transport phenomena. Wiley, New York, pp 582-606

42. Zhang D, Zhang X, Wei J, Gu G, Xiang G (2015) Growth of tapered silica nanowires with a shallow U-shaped vapor chamber: 
growth mechanism and structural and optical properties. J Appl Phys 117:164303. https://doi.org/10.1063/1.4918718

43. Smith AD, Elgammal K, Niklaus F, Delin A, Fischer AC, Vaziri SV, Forsberg $\mathrm{F}$, Råsander $\mathrm{M}$, Hugosson $\mathrm{H}$, Bergqvist L, Schröder $\mathrm{S}$, Kataria S, Östlinga M, Lemme MC (2015) Resistive graphene humidity sensors with rapid and direct electrical readout. Nanoscale 7:19099-19109. https://doi.org/10.1039/c5nr06038a
44. Annanouch FE, Bouchet G, Perrier P, Morati N, Reynard-Carette C, Aguir K, Martini-Laithier V, Bendahan M (2019) Hydrodynamic evaluation of gas testing chamber: simulation, experiment. Sens Actuators B Chem 290:598-606. https://doi.org/10.1016/j. snb.2019.04.023

Publisher's Note Springer Nature remains neutral with regard to jurisdictional claims in published maps and institutional affiliations. 